\title{
DIAGNOSIS OF TUBERCULOSIS LYMPHADENITIS USING A POLYMERASE CHAIN REACTION ON PERIPHERAL BLOOD MONONUCLEAR CELLS
}

\author{
SHAPER MIRZA, BLANCA I. RESTREPO, JOSEPH B. MCCORMICK, AND SUSAN P. FISHER-HOCH \\ Department of Pathology, Aga Khan Hospital, Karachi, Pakistan; University of Texas Health Science Center Houston School of \\ Public Health, Brownsville, Texas
}

\begin{abstract}
Providing prompt and precise laboratory confirmation of a clinical diagnosis of tuberculous lymphadenitis is difficult given the paucibacillary nature of lymph node specimens. In this study carried out in Karachi, Pakistan, a polymerase chain reaction (PCR) assay aimed at detecting Mycobacterium tuberculosis DNA in peripheral blood mononuclear cells (PBMC-PCR) was standardized and compared with standard $M$. tuberculosis diagnostic techniques or a lymph node PCR (LN-PCR) for the diagnosis of tuberculosis lymphadenitis. Thirty-seven (77\%) specimens from 48 patients with clinical or diagnosis of tuberculosis lymphadenitis were positive by cytology [17/48 (35\%) with no acid fast bacilli (AFB) (suggestive), and 20/48 (42\%) with AFB (positive) in direct smears], 30 (63\%) by PBMC-PCR, 16 (33\%) by LN-PCR, and $13(27 \%)$ by culture. All controls were negative, with the exception of one false-positive LN-PCR. These data suggest the PBMC-PCR may be helpful in the diagnosis of tuberculous lymphadenitis.
\end{abstract}

\section{INTRODUCTION}

Tuberculosis (TB) is on the rise globally with an estimated one third of the world's population being infected with $M y$ cobacterium tuberculosis, and approximately 3-4 million new cases per year. Developing countries, particularly those in southeast Asia, have experienced a major increase in the burden of TB disease, a problem that is compounded by the emergence of multi-drug resistant TB. ${ }^{1}$ Pakistan is one of the 22 countries with the greatest incidence of TB morbidity, with an estimated 247,000 cases in year 2000 , among whom 111,000 were sputum smear positive. ${ }^{1}$ Extra-pulmonary TB is higher in Asian than in Caucasian populations., ${ }^{2,3}$ Tuberculous lymphadenitis (TB-L) is the most common presentation of extrapulmonary $\mathrm{TB}$, accounting for $30-40 \%$ of cases in reported series. ${ }^{4}$ Although there are no data available on the prevalence of extra-pulmonary tuberculosis in Pakistan, 58\% of specimens received for $M$. tuberculosis culture and/or polymerase chain reaction (PCR) at the Aga Khan University Hospital laboratory in Karachi are from extra-pulmonary sites, with lymph node specimens being the most common.

In developing countries the detection of TB-L with traditional diagnostic tools is a major challenge. Direct smears are widely used but lack sensitivity and specificity. Culture takes at least two weeks, is not always available in countries endemic for TB, and lacks sensitivity in extra-pulmonary TB. Subsequent drug sensitivity testing takes $2-3$ additional weeks. ${ }^{5}$ The diagnosis of TB-L is routinely performed by lymph node cytology or biopsy histology. However, histology and cytology lack specificity due to the difficulty of distinguishing other granulomatous pathologies in the absence of acid-fast bacilli, and the process of biopsy is invasive.

The PCR has been evaluated for more than a decade for the diagnosis of pulmonary and extrapulmonary TB, including TB-L. ${ }^{6-9}$ Its development has required extensive troubleshooting to avoid false-positive and false-negative results, and reproducibility between laboratories. However, it is now a recognized approach in the routine laboratory setting and commercial kits are available to complement conventional diagnostic assays. ${ }^{10,11}$ The PCR is routinely used in specimens from the site of infection, such as sputum for pulmonary TB, cerebrospinal fluid for tuberculous meningitis, and lymph node material for TB-L. An alternative approach is the de- tection of $M$. tuberculosis or $M$. avium DNA with a PCR in peripheral blood mononuclear cells (PBMC-PCR) of patients with pulmonary TB. ${ }^{12-17}$ The hypothesis is that patients with active infection harbor mycobacterial DNA in peripheral scavenging cells such as macrophages. ${ }^{13,15}$ In TB patients coinfected with human immunodeficiency virus (HIV), the PCR has sensitivities of $95-100 \%$ and specificities of 89-100\%. ${ }^{12-14,17}$ In case series with immunocompetent and immunosuppressed patients (HIV negative), the performance of the assay is highly variable, ranging from $33 \%$ to $87 \%$ depending on the study. ${ }^{15,16,18-20}$ The use of the PBMC-PCR for the diagnosis of extrapulmonary TB is still incipient but holds promise. ${ }^{16,21}$

Our study was performed at the Aga Khan University, with an affiliated 650 bed modern, private hospital, the Aga Khan Hospital, in Karachi, Pakistan. It has one of the most modern and well-equipped clinical laboratories in the country and provides rapid and timely histologic and microbiologic diagnostic services throughout Pakistan. One of the daily challenges in the clinical laboratory is to provide an accurate diagnosis of TB-L, the most common form of extrapulmonary TB. In this study, we sought to develop and evaluate a PBMCPCR assay in patients with a clinical suspicion of TB-L.

\section{MATERIALS AND METHODS}

Patient selection. Patients consecutively referred to the Aga Khan Hospital Clinical Laboratory for analysis by fine needle aspiration cytology (FNAC) to confirm the clinical suspicion of TB-L were invited to participate. Clinical suspicion was based on chronic, low-grade fever, weight loss, anorexia, and enlarged palpable lymph nodes. Control patients with lymph node pathology usually had clinical suspicion of neoplasm and were referred for biopsy of solid tumors. Since non-caseous nodes cannot be aspirated, biopsy samples were obtained from these nodes. A second control group for the consisted of 40 healthy individuals. None of the patients or controls had evidence of pulmonary TB. Infection with HIV was not examined in the patients or controls, but none presented signs or symptoms associated with acquired immunodeficiency syndrome (AIDS), and HIV prevalence in Pakistan is and was extremely low. The study was reviewed and 
approved by the Internal Review Committee of the Aga Khan Hospital Medical School in Karachi. All patients in this study were middle or high income residents of Karachi.

Sampling techniques and specimen handling. The FNAC was performed with the palpable lymph nodes of suspected cases by an experienced pathologist as an outpatient procedure. Drops of the aspirate material were immediately expressed and spread on glass slides, fixed, and stained with Papanicolaou stain, hematoxylin and eosin, and ZiehlNeelsen stain. The material remaining in the syringe was used directly for $M$. tuberculosis culture or a lymph node PCR (LN-PCR). Similarly, the biopsy specimens from control patients were processed for histologic analysis, direct smear, $M$. tuberculosis culture, and LN-PCR. For histology, an aliquot of the specimen was fixed in $10 \%$ buffered formalin, and 8 -mm sections processed in an automated tissue processor for 12 hours, embedded in paraffin, and stained with hematoxylin and eosin and Ziehl-Neelsen stain. A section of the biopsy was homogenized used for M. tuberculosis culture and LN-PCR analysis. Cytology smears and tissue sections stained with Papanicolaou stain, hematoxylin and eosin, and ZiehlNeelsen stain were screened by experienced cytotechnologists and results were recorded as suggestive TB-L if there was typical caseating necrosis in the absence of acid fast bacilli or positive TB-L if acid fast bacilli were detected by ZiehlNeelsen stain. Culture for M. tuberculosis was performed using the Bactec 460 radiometric system (Becton Dickinson, Franklin Lakes, NJ) by incubation at $37^{\circ} \mathrm{C}$ for six weeks or until a positive reaction was observed. A $10-\mathrm{mL}$ blood specimen was collected in heparinized tubes from all patients and controls for PBMC-PCR analysis. The buffy coat from the heparinized blood was separated using Ficoll Hypaque (density 1.007 grams/ml; Sigma, St. Louis, MO) and PBMCs were collected for PCR.

PCR assays. Extraction of DNA from PBMCs, lymph node FNAC samples, and biopsy specimens was performed as described previously. ${ }^{22}$ Essentially, cell walls were lysed with lysozyme, followed by proteinase $\mathrm{K}$ digestion and sodium dodecyl sulfate treatment of proteins. Proteins and macromolecules were precipitated using $\mathrm{NaCl}$ and hexadecyltrimethlyammonium bromide $/ \mathrm{NaCl}$ solutions. Nucleic acids were recovered from the aqueous phase after extraction with chloroform and isoamyl alcohol. The DNA was precipitated overnight with isopropanol at $-20^{\circ} \mathrm{C}$. The pellet was washed with ethanol, reconstituted in $50 \mu \mathrm{L}$ of water, and $10 \mu \mathrm{L}$ were used in the PCR. Amplification of the DNA was performed with the primers INS-1 5'-CGTGAGGGCATCGAGGTGGC-3' and INS-2 5'-GCGTAGGCGTCGGTGACAAA-3', which amplify a 245-basepair segment of the insertion element IS6110. ${ }^{23}$ Because some of the M. tuberculosis isolates in Pakistan do not carry this insertion element, ${ }^{24} \mathrm{a}$ second set of primers targeting a 439-basepair fragment of the $65-\mathrm{kD}$ heat shock protein from all mycobacterial species was also tested in all specimens. The primer sequences were TB11 5'-ACCAACGATGGTGTGTCCAT-3' and TB12 5'CTTGTCGAACCGCATACCCT- $3{ }^{\prime}{ }^{25}$ The PCR was performed using $10 \mathrm{mM}$ Tris, $50 \mathrm{mM} \mathrm{KCl}, 1.5 \mathrm{mM}$ of each dNTP, $0.4 \mu \mathrm{M}$ of each primer, and 2.5 units of Taq polymerase. Cycling conditions were denaturation at $94^{\circ} \mathrm{C}$ for two minutes, annealing at $65^{\circ} \mathrm{C}$ for two minutes, and extension at $72^{\circ} \mathrm{C}$ for three minutes, for a total of 40 cycles. The amplified products were subjected to electrophoresis on a $1.5 \%$ agarose gel and a positive PCR was evaluated by the detection of the expected amplicons after staining with $0.5 \mu \mathrm{g} / \mathrm{ml}$ of ethidium bromide.

\section{RESULTS}

Forty-seven cases were referred for FNAC for confirmation of TB-L by lymph node aspiration, and nine controls with enlarged, solid lymph nodes underwent a biopsy as part of their routine medical evaluation for lymph node carcinoma. For ethical reasons, obtaining lymph node material from healthy controls was not possible, except in these nine patients already undergoing biopsies as part of their routine medical evaluation for lymph node carcinoma. One of these control patients was an 80-year-old woman with biopsyproven squamous cell carcinoma. However, her lymph node biopsy was also positive for M. tuberculosis by culture, $\mathrm{LN}$ PCR assays, and PBMC-PCR. Accordingly, she was reallocated to the TB-L patient group (total TB-L $=48$, controls $=$ 8 ). The patients with non-TB-L were significantly older (median $=50$ years old, range $=8-80$ ) than those with TB-L (median $=20$ years old, range $=8-80)(P=0.001$, by Kruskal-Wallis test). The male to female ratio was 1:2.4 among patients with TB-L and 1:1 in the non-TB-L group. To control for this age difference, a second healthy control group of young adults was bled for PBMC-PCR analysis.

Using a clinical diagnosis of TB-L as a reference, individual analysis of each assay indicated, in order of sensitivity, that the combined positive and suggestive cytology was positive in $77 \%$ of the cases, including 17 (35\%) of 48 suggestive (granuloma with negative direct smear for M. tuberculosis) and 20 $(42 \%)$ of 48 positive (granuloma with positive direct smear for M. tuberculosis) cytology (Figure 1). The second highest sensitive assay was the PBMC-PCR, which was positive in 30 patients $(62.5 \%)$, with identical results with either the IS6110 and hsp65 targets. Finally, the LN-PCR was positive in 16 (33\%) cases, and M. tuberculosis was isolated from $13(27 \%)$

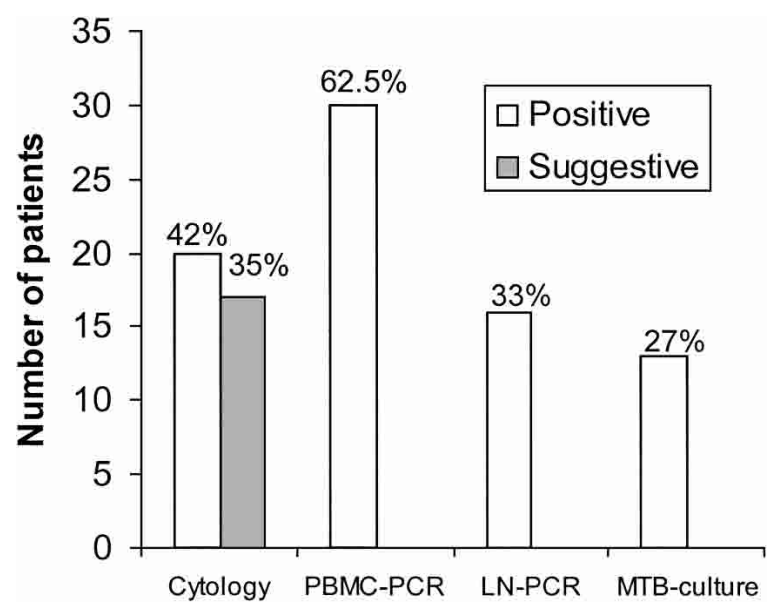

FIGURE 1. Number of patients with clinically diagnosed tuberculous lymphadenitis (TB-L) that were positive by each assay. The 48 patients with a clinical diagnosis of TB-L were examined by fine needle aspiration cytology (gray bar $=$ suggestive, open bars $=$ positive, based on the detection of acid-fast bacilli), peripheral blood mononuclear cell-polymerase chain reaction (PBMC-PCR), lymph node PCR (LN-PCR), and M. tuberculosis (MTB) culture. Percent positive are indicated above each bar. 
(Figure 1). In the clinical setting, a positive culture is the gold standard for diagnosis (Figures 1 and 2). The next most reliable laboratory results are the combination of culture and direct smear (reported as positive cytology), which increased the sensitivity to $56 \%$ (Figure 2). Culture and smear are routinely complemented with cytology (positive and suggestive), increasing the overall sensitivity to $81 \%$ in this study. However, $12(25 \%)$ of these cases had a suggestive cytology as the only laboratory evidence for TB-L. The additional contribution of the PBMC-PCR to the routine combination of assays confirmed the suggestive cytology result in nine of these 12 cases, and identified three new patients who were negative by all other assays. Thus, with the addition of the PBMC-PCR, there were $39(81.25 \%)$ positive and $3(6.25 \%)$ suggestive cases, increasing the overall sensitivity to $87.5 \%$ (Figure 2). The two assays that provided the highest combined sensitivity were the PBMC-PCR and cytology, which identified 85\% (41 of 48) of all clinically suspected cases of TB-L. These two assays were concordant in $68.8 \%$ of the cases ( 26 positive and 7 negative). One of the eight control, non-TB-L patients (based on clinical criteria) had a positive result in the LNPCR. The results of all other assays were negative in the control patients.

\section{DISCUSSION}

Despite the continuing difficulty in providing prompt and satisfactory laboratory confirmation of a clinical diagnosis for TB-L, we have shown that detection of M. tuberculosis DNA in circulating mononuclear blood cells holds promise as a rapid screening assay and an adjunct to routine techniques. The PBMC-PCR alone was positive in $65.2 \%$ of the cases

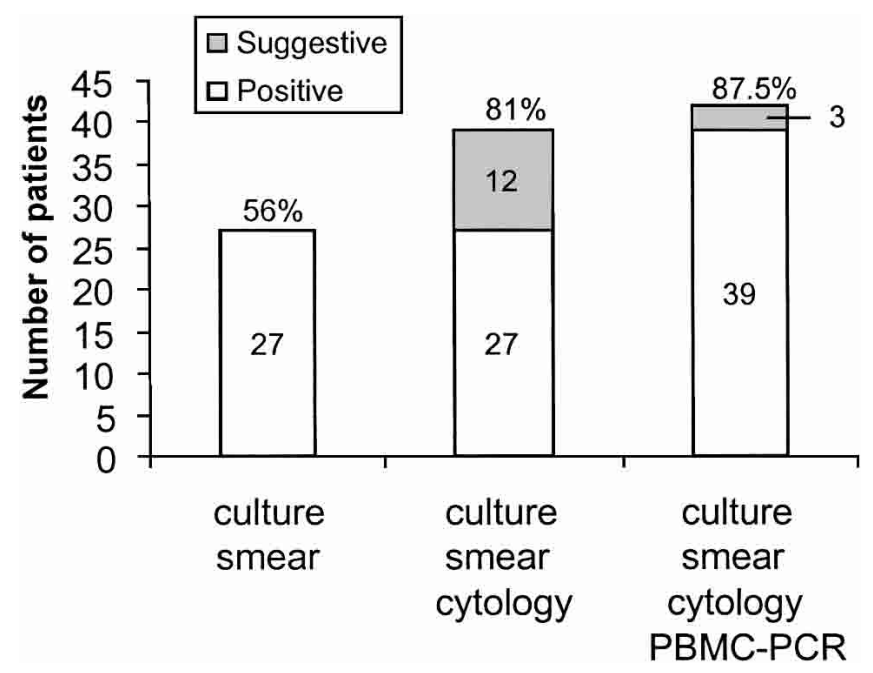

FIGURE 2. Contribution of combined assays for a diagnosis of tuberculous lymphadenitis (TB-L). Combined Mycobacterium tuberculosis culture and direct smear were positive in 27 cases $(56 \%)$. In the routine clinical setting, culture and direct smear are complemented with cytology, providing a diagnosis for 39 cases $(81 \%)$, from which $12(25 \%)$ only had a suggested cytology as supporting evidence for diagnosis. If the routine laboratory assays are complemented with a peripheral blood mononuclear cell-polymerase chain reaction (PBMC-PCR), the diagnosis is made in 42 (87.5\%) of 48 patients, and the number of positives with a suggestive cytology alone is reduced to three cases $(6.25 \%)$. Gray bars = suggestive TB-L; open bars $=$ positive TB-L. with clinical suspicion of TB-L and in none of the controls. In the absence of an optimal gold standard that confirms the clinical suspicion of TB-L, and since the controls were all negative, the data suggested that the PBMC-PCR is highly sensitive when compared with conventional techniques, and is more specific than cytologic examination.

The onset of TB-L is insidious. Patients present with enlarged, usually painless lymph nodes, most commonly affecting the cervical region. Systemic symptoms such as fever, weight loss, and malaise may or may not be present at an early stage. Advanced disease presents additionally with cold abscess with or without sinus formation. Prompt diagnosis and effective treatment leads to reduced morbidity and mortality. To obtain early and accurate diagnosis, rapid laboratory techniques are required. Classic methods based on the detection of $M$. tuberculosis at the site of infection have low sensitivity. Our results using these established methods bear this out and fall within the range of those reported in the literature. ZiehlNeelsen staining is rapid and inexpensive, but requires more than 10,000 organisms/mL, cannot be used to distinguish between various members of mycobacteria, and its sensitivity ranges between $10 \%$ and $50 \%$ depending on the study. ${ }^{7,8,26,27}$ In our study, acid-fast bacilli were detected in $42 \%$ of the cases (reported as positive TB-L cytology). Culture is the gold standard, but takes several weeks, requires 10-100 organisms per sample, and reports from different studies detect in fine needle aspirates or biopsy specimens between $9 \%$ and $70 \%$ positive in the clinically suspected TB-L cases. ${ }^{7,8,26,27}$ In our study, $27 \%$ of the fine needle aspirates from probable TB-L patients were positive. The recognized poor sensitivity of conventional microbiologic assays ${ }^{7,8}$ is routinely complemented with additional non-specific assays. In FNAC or histology of surgical lymph node biopsy specimens, diagnosis is based on the presence of granulomas, central necrosis (suggestive TBL), and if possible, demonstration of acid-fast bacilli by staining of tissue sections (positive TB-L). However, histology lacks specificity since granulomas with necrosis are seen in several infectious and non-infectious conditions, and falsenegative Ziehl-Neelsen smears are common (presumably $35 \%$ in our study assuming all suggestive cytologies were indeed TB-L). Histology and cytology have a sensitivity of approximately $32-59 \%$, depending on the report, ${ }^{6,8,27}$ and were $77 \%$ positive (combined positive and suggestive) in our hands.

The PCR has gained importance in the clinical diagnosis of M. tuberculosis, and is now an important support in the clinical diagnosis of TB in developed countries. ${ }^{11}$ Despite its considerable demands for technical skills and equipment, it can also be performed successfully in reference laboratories in developing countries, such as the modern Aga Khan clinical laboratory in Karachi, Pakistan. ${ }^{24}$ Given the availability of a thermal cycler, the rest of the procedure has a cost similar to other routine assays for TB-L diagnosis. Additional advantages of PCR over conventional methods include its high sensitivity, performance in few hours, and depending on the assay design, ability to differentiate between $M$. tuberculosis complex and mycobacterial species other than TB, and identification of gene mutations associated with drug resistance. ${ }^{28,29}$ In the past decade, experimental studies describing lymph node PCRs from fine needle aspirates or biopsy specimens have consistently shown improved sensitivity (61-78\% depending on the study) when compared with conventional 
microbiologic methods. ${ }^{6-8}$ In the present study, the LN-PCR was positive in $33 \%$ of the aspirates from patients. This is a lower sensitivity compared with other reports for TB-L patients, perhaps due to the small amount of aspirate remaining after splitting the specimen for the additional microbiologic and cytologic assays.

In one study, comparative analysis showed biopsies had higher sensitivity than aspirates, presumably due to their larger size. ${ }^{8}$ However, in the routine clinical setting, aspirates are preferred because their collection is less invasive. At the Aga Khan Hospital, aspirates were routinely performed for TB-L diagnosis, while biopsies were performed for histologic analysis of patients with suspected malignancies. This difference in specimen collection between patients and controls was done because aspirates cannot be performed on solid tumors, and it was not justified to perform biopsies, a more invasive procedure, on patients with caseous lymph nodes. If this aspect of our experimental design affected our results, one would expect biopsies to provide higher sensitivity for $M$. tuberculosis detection given their larger size compared with aspirates. One of the biopsy samples gave a positive LN-PCR result in the control specimens, but the interpretation of this unexpected result is unclear.

The working hypothesis for the PBMC-PCR is that peripheral macrophages have scavenged $M$. tuberculosis genomic material from the infected sites, or even whole bacilli. The detection of $M$. tuberculosis DNA, but our inability to obtain culture-positive blood from the same individuals, suggested low-level bacteremia undetectable by culture, or most likely, amplification of DNA remnants from non-viable mycobacteria, presumably inside phagocytic vacuoles from peripheral macrophages.

We had difficulty obtaining a larger number of lymph node and blood specimens from patients who did not have a clinical suspicion of TB-L, and those sampled were significantly older than the TB patients. To control for this age difference, blood samples for the PBMC-PCR were collected from 40 younger, normal, healthy adults, all of whom were negative. Even though a tuberculin skin test was not performed, the fact that the controls from a population that is highly endemic for TB were negative suggested that a positive PBMC-PCR result is usually associated with active TB infection. Likewise, the HIV or AIDS status of the patients or controls was not available. This infection is thought to be essential in increasing the sensitivity of blood-based PCR assays for pulmonary TB. ${ }^{12-14,17}$ However, it is unlikely that the patients had AIDS because none presented signs or symptoms suggestive of AIDS, and the prevalence of HIV (0.007\%) and AIDS (1,710 cases/140 million population) is extremely low in Pakistan. ${ }^{30}$ These data suggested that the PBMC-PCR can provide a high sensitivity for the diagnosis of TB-L patients in non-AIDS populations.

Our results, combined with findings from a previous study, ${ }^{16,21}$ encourage us to test the PBMC-PCR in the diagnosis of other forms of extrapulmonary TB where the infections are usually paucibacillary, have an extensive differential diagnosis, and access to specimens requires an invasive procedure. As the PCR technology improves, new advances allowing real time assays, high sensitivity and specificity, and high throughput at low cost open up improved possibilities for diagnosis.
Received April 16, 2003. Accepted for publication July 14, 2003.

Acknowledgments: We thank the field staff of the Urban Health Program for their help during data collection, and the staff of the research laboratories of Aga Khan University for their participation in the study.

Financial support: This project was supported in part by the Aga Khan Health Services of Pakistan.

Authors' addresses: Shaper Mirza, Department of Microbiology, University of Alabama, Birmingham, Bevill Building, 845 19th South Street, Birmingham, AL 35294, Telephone: 205-934-1923, Fax: 205934-0605. Blanca I. Restrepo, Joseph B. McCormick, and Susan P. Fisher-Hoch, University of Texas Health Science Center Houston School of Public Health-Brownsville, The University of Texas at Brownsville, 80 Fort Brown SPH, 2nd Floor Room 2.202, Brownsville, TX 78520.

Reprint requests: Blanca I. Restrepo, University of Texas Health Science Center Houston School of Public Health, The University of Texas at Brownsville, 80 Fort Brown, SPH 2nd Floor, Room 2.202 Brownsville, TX 78520, Telephone: 956-279-3841, Fax: 956-554-5152, E-mail: birestrepo@utb.edu.

\section{REFERENCES}

1. WHO. 2002. WHO Report 2002: Global Tuberculosis Control. WHO. http://www.who.int/gtb/publications/globrep02/ downloadpage.html

2. Styblo K, 1990. The elimination of tuberculosis in The Netherlands. Bull Int Union Tuberc Lung Dis 65: 49-55.

3. Finch PJ, Millard FJ, Maxwell JD, 1991. Risk of tuberculosis in immigrant Asians: culturally acquired immunodeficiency? Thorax 46: 1-5.

4. Hooper AA, 1972. Tuberculous peripheral lymphadenitis. $\mathrm{Br}$ J Surg 59: 353-359.

5. Small PM, Moss A, 1993. Molecular epidemiology and the new tuberculosis. Infect Agents Dis 2: 132-138.

6. Baek CH, Kim SI, Ko YH, Chu KC, 2000. Polymerase chain reaction detection of Mycobacterium tuberculosis from fineneedle aspirate for the diagnosis of cervical tuberculous lymphadenitis. Laryngoscope 110: 30-34.

7. Gong G, Lee H, Kang GH, Shim YH, Huh J, Khang SK, 2002. Nested PCR for diagnosis of tuberculous lymphadenitis and PCR-SSCP for identification of rifampicin resistance in fineneedle aspirates. Diagn Cytopathol 26: 228-231.

8. Singh KK, Muralidhar M, Kumar A, Chattopadhyaya TK, Kapila K, Singh MK, Sharma SK, Jain NK, Tyagi JS, 2000. Comparison of in house polymerase chain reaction with conventional techniques for the detection of Mycobacterium tuberculosis DNA in granulomatous lymphadenopathy. J Clin Pathol 53: 355-361.

9. Narita M, Shibata M, Togashi T, Kobayashi H, 1992. Polymerase chain reaction for detection of Mycobacterium tuberculosis. Acta Paediatr 81: 141-144.

10. CDC, 2000. Update: Nucleic acid amplification tests for tuberculosis. MMWR Morb Mortal Wkly Rep 49: 593-594.

11. Brown TJ, Power EG, French GL, 1999. Evaluation of three commercial detection systems for Mycobacterium tuberculosis where clinical diagnosis is difficult. J Clin Pathol 52: 193-197.

12. Condos R, McClune A, Rom WN, Schluger NW, 1996. Peripheral-blood-based PCR assay to identify patients with active pulmonary tuberculosis. Lancet 347: 1082-1085.

13. Schluger NW, Condos R, Lewis S, Rom WN, 1994. Amplification of DNA of Mycobacterium tuberculosis from peripheral blood of patients with pulmonary tuberculosis. Lancet 344: 232-233.

14. De Francesco MA, Colombrita D, Pinsi G, Gargiulo F, Caligaris S, Bertelli D, Martinelli F, Gao J, Turano A, 1996. Detection and identification of Mycobacterium avium in the blood of AIDS patients by the polymerase chain reaction. Eur J Clin Microbiol Infect Dis 15: 551-555.

15. Del Prete R, Mosca A, D'Alagni M, Sabato R, Picca V, Miragliotta G, 1997. Detection of Mycobacterium tuberculosis DNA in blood of patients with acute pulmonary tuberculosis 
by polymerase chain reaction and non-isotopic hybridization assay. J Med Microbiol 46: 495-500.

16. Honore S, Vincensini JP, Hocqueloux L, Noguera ME, Farge D, Lagrange P, Herrmann JL, 2001. Diagnostic value of a nested polymerase chain reaction assay on peripheral blood mononuclear cells from patients with pulmonary and extrapulmonary tuberculosis. Int J Tuberc Lung Dis 5: 754-762.

17. Kulski JK, Khinsoe C, Pryce T, Christiansen K, 1995. Use of a multiplex PCR to detect and identify Mycobacterium avium and $M$. intracellulare in blood culture fluids of AIDS patients. J Clin Microbiol 33: 668-674.

18. Aguado JM, Rebollo MJ, Palenque E, Folgueria L, 1996. Bloodbased PCR assay to detect pulmonary tuberculosis. Lancet 347 : 1836-1837.

19. Ahmed N, Mohanty AK, Mukhopadhyay U, Batish VK, Grover S, 1998. PCR-based rapid detection of Mycobacterium tuberculosis in blood from immunocompetent patients with pulmonary tuberculosis. J Clin Microbiol 36: 3094-3095.

20. Rolfs A, Beige J, Finckh U, Kohler B, Schaberg T, Lokies J, Lode H, 1995. Amplification of Mycobacterium tuberculosis from peripheral blood. J Clin Microbiol 33: 3312-3314.

21. Tzoanopoulos D, Stakos D, Hatseras D, Ritis K, Kartalis G, 2001. Detection of Mycobacterium tuberculosis complex DNA in pericardial fluid, bone marrow and peripheral blood in a patient with pericardial tuberculosis. A case report. Neth J Med 59: $177-180$

22. Ausubel FM, Brent R, Kingston RE, Moore DD, Seidman JG, Smith JA, Struhl K, 1998. Preparation of genomic DNA from bacteria. Current Protocols in Molecular Biology. New York: John Wiley \& Sons, 1129-1215.
23. Hermans PW, Schuitema AR, van Soolingen D, Verstynen CP, Bik EM, Thole JE, Kolk AH, van Embden JD, 1990. Specific detection of Mycobacterium tuberculosis complex strains by polymerase chain reaction. J Clin Microbiol 28: 1204-1213.

24. Moatter T, Mirza S, Siddiqui MS, Soomro IN, 1998. Detection of Mycobacterium tuberculosis in paraffin embedded intestinal tissue specimens by polymerase chain reaction: characterization of IS6110 element negative strains. J Pak Med Assoc 48: 174-178.

25. Telenti A, Marchesi F, Balz M, Bally F, Bottger EC, Bodmer T, 1993. Rapid identification of mycobacteria to the species level by polymerase chain reaction and restriction enzyme analysis. J Clin Microbiol 31: 175-178.

26. Huhti E, Brander E, Paloheimo S, Sutinen S, 1975. Tuberculosis of the cervical lymph nodes: a clinical, pathological and bacteriological study. Tubercle 56: 27-36.

27. Kim SS, Chung SM, Kim JN, Lee MA, Ha EH, 1996. Application of PCR from the fine needle aspirates for the diagnosis of cervical tuberculous lymphadenitis. J Korean Med Sci 11: 127132.

28. Piatek AS, Tyagi S, Pol AC, Telenti A, Miller LP, Kramer FR, Alland D, 1998. Molecular beacon sequence analysis for detecting drug resistance in Mycobacterium tuberculosis. Nat Biotechnol 16: 359-363.

29. Richeldi L, Barnini S, Saltini C, 1995. Molecular diagnosis of tuberculosis. Eur Respir J 8: 689s-700s.

30. Khattak MF, 2002. Salamat N, Bhatti F A, Qureshi T Z. Seroprevalence of hepatitis B, C and HIV in blood donors in northern Pakistan. J Pak Med Assoc 52: 398-402. 